\title{
AGRUPAMENTO DE DADOS MISTOS BASEADOS NO ALGORITMO K-MEANS: UMA REVISÃO SISTEMÁTICA DA LITERATURA
}

\author{
Nádia Junqueira Martarelli e Marcelo Seido Nagano \\ Departamento de Engenharia de Produção, Escola de Engenharia de São Carlos, Universidade de São Paulo \\ Avenida Trabalhador São-Carlensce n. 400, São Carlos, Brasil. CEP 13566-590
}

\begin{abstract}
RESUMO
O agrupamento de dados é uma atividade de mineração que busca por grupos naturais em conjuntos de dados não rotulados. Embora bem desenvolvida para dados numéricos, o desenvolvimento de algoritmos para dados mistos (atributos numéricos e categóricos) ainda é incipiente. Diante do fato de que os conjunto de dados mistos caracterizam a maioria dos problemas reais, o avanço desta temática é essencial para o beneficiamento da sociedade e da indústria. Dessa forma, este trabalho tem como objetivo reunir e discutir as principais contribuições publicadas na literatura sobre algoritmos de agrupamento de dados mistos, especificamente as abordagens baseadas no algoritmo $k$-means, já que este algoritmo apresenta alta velocidade de processamento e fácil implementação, características estas almejadas na resolução de problemas do mundo real. Diante disso, realizou-se uma revisão bibliográfica sistemática na base de dados Scopus, onde foram aceitos apenas artigos em inglês, com o indicador Jornal Citation Report (JCR) maior ou igual a um, ou com pelo menos dois indicadores de impacto maior ou igual a um. Como primeiro resultado da busca, obteve-se 1.104 trabalhos. Após a aplicação dos critérios adotados, obteve-se 456 trabalhos passíveis de avaliação. Posteriormente, avaliou-se quais trabalhos estavam dentro do objetivo da análise, por meio de três filtros de seleção, os quais contemplaram diferentes leituras dos trabalhos. Como resultado, obteve-se 37 artigos. Após um estudo detalhado, observou-se que esta temática ainda apresenta um desenvolvimento recente e esparso. Além disso, percebeu-se que os trabalhos selecionados exploraram alguns temas-chave, como a definição dos pesos dos atributos, número de grupos, inicialização dos centroides e agrupamento sujeito a restrições.
\end{abstract}

\section{PALAVRAS-CHAVE}

Agrupamento de Dados Mistos, K-means, Atributos Numéricos e Categóricos, Clustering, Revisão Sistemática

\section{INTRODUÇÃO}

A técnica de agrupamento de dados é uma tarefa de mineração que busca por grupos naturais em conjuntos de dados não rotulados (Jain and Dubes, 1988). Por desvendar padrões em dados que não apresentam nenhuma classificação prévia, esta tarefa é considerada uma das mais desafiadoras dentre as técnicas de reconhecimento de padrões. Embora desafiadora, os algoritmos de agrupamento de dados são altamente demandados por inúmeras áreas do conhecimento, para os mais diferentes propósitos, como a segmentação de clientes, diagnóstico de doenças, classificação de estrelas e planetas, análise de tendência mercadológica, definição de taxonomias, identificação de genes e proteínas, reconhecimento biométrico, segmentação de imagens, análise do padrão de comportamento, entre muitas outras aplicações (Xu and Wunsch, 2009).

Com o surgimento de uma ampla gama de algoritmos de agrupamento de dados, surgiu a necessidade didática e prática de classificá-los. Uma das classificações mais aceitas e disseminadas consideram como característica segregadora a forma com que os grupos são encontrados pelos algoritmos. Assim, a literatura apresenta quatro principais classes de algoritmos de agrupamento de dados, $(i)$ os particionais, que separam os registros do conjunto de dados em $k$ grupos por uma medida de similaridade ou dissimilaridade, (ii) os hierárquicos, que encontram todos os possíveis grupos para posteriormente selecionar um deles, (iii) os baseados em modelo, que adotam como premissa que dados seguem distribuições estatísticas, e (iv) os baseados em redes neurais, que encontram o grupos aplicado os conceitos de redes neurais no conjunto de dados (Xu and Wunsch, 2009). 
Apesar de cada tipo de algoritmo apresentar vantagens e desvantagens, não é possível afirmar que há soberania entre eles, independentemente do problema, o que significa que cada um responde de maneira específica às características do conjunto de dados e, principalmente, ao objetivo da análise (Jain, 2010). No entanto, os algoritmos particionais são, de maneira geral, mais utilizados do que outros em problemas reais, por apresentarem características que favorecem suas aplicações, como a facilidade de implementação, a linearidade em relação ao número de registros do conjunto de dados e o rápido processamento (Ahmad and Khan, 2019). Um destes algoritmos é o k-means, um dos primeiros algoritmos de agrupamento de dados particional, que utiliza a distância Euclidiana para associar cada registro do conjunto de dados a um dos $k$ grupos pré-definidos pelo usuário. O k-means, que foi proposto por MacQueen (1967), aceita apenas conjuntos de dados numéricos, assim como a maioria dos algoritmos de agrupamento de dados disponíveis atualmente.

Embora haja um avançado desenvolvimento dos algoritmos de agrupamento de dados para dados de tipo único, esta não é a realidade da maioria dos conjuntos de dados dos problemas reais, já que em muitos casos os registros são caracterizados por atributos mistos (dados numéricos e categóricos). Um exemplo simples de conjunto de dados mistos em um problema real é uma base de dados de clientes. Para realizar a agrupamento de clientes por um algoritmo de agrupamento de dados, este deve ser capaz de lidar com atributos numéricos (idade e média salarial, por exemplo) e atributos categóricos (sexo, cidade, estado, país etc.).

Duas estratégias têm sido adotadas para lidar com estes tipos de dados. A primeira consiste em transformar o conjunto de dados mistos em um conjunto de dados de tipo único, utilizando algum método de transformação de dados. A segunda estratégia consiste em calcular a dissimilaridade de ambos os tipos de dados, na mesma função objetivo (Foss, Markatou and Ray, 2019). Ainda que a transformação dos dados (primeira estratégia) possa usufruir do desenvolvimento dos algoritmos de tipo único, este procedimento muda o espaço original dos dados, podendo torná-los menos inteligíveis. Ao considerar duas funções de dissimilaridade na mesma função objetivo (segunda estratégia), os dados originais são mantidos, mas pelo fato do desenvolvimento este tipo de algoritmo ainda ser recente, eles podem ser menos eficientes do que os algoritmos que lidam apenas com dados numéricos ou categóricos, além de apresentarem alguns desafios técnicos e conceituais, que atualmente se mostram como questões abertas de pesquisa, as quais foram sumarizadas em alguns artigos de revisão.

Em 2018, dois artigos de revisão foram publicados. O trabalho de Balaji and Lavanya (2018) objetivou realizar uma crítica análise dos algoritmos mais efetivos para realizar o agrupamento de dados mistos. Embora os autores tenham descrito os trabalhos considerados com atributos relevantes para a análise, eles se limitaram a citar poucos algoritmos de cada tipo de agrupamento. Miyamoto, Huynh and Fujiwara (2018) trouxeram uma suscita apresentação de algumas medidas de dissimilaridade usadas para dados categóricos e fizeram uma breve descrição de poucos algoritmos. Por isso, o trabalho se resume em um detalhamento superficial que não explora profundamente a temática. Em 2019, mais três artigos de revisão foram publicados. van de Velden, D'Enza and Markos (2019) propuseram uma revisão dos algoritmos de agrupamento de dados baseados em distância, especificamente os algoritmos particionais e hierárquicos, abordando como a redução de dimensionalidade é realizada para os conjuntos de dados mistos e apontando as funções de dissimilaridade mais utilizadas. Os autores também compararam cinco algoritmos de agrupamento de dados mistos em três bases de dados. Embora o artigo tenha proposto uma discussão sobre a temática em questão, os autores exploraram superficialmente os tópicos, deixando de citar trabalhos importantes da área e se limitando a apresentar as abordagens principais, sem realizar uma discussão e sumarização consistente do tema. Foss, Markatou and Ray (2019) apresentaram uma revisão sobre as medidas de distância e os métodos de agrupamento para dados mistos, analisando de maneira teórica e empírica os trabalhos considerados, os quais se referiam apenas aos algoritmos particionais e baseados em modelo. Ahmad and Khan (2019) trouxeram uma extensa e completa revisão sobre o agrupamento de dados mistos, citando uma ampla gama de trabalhos nas diversas classes de agrupamento. Além disso, o trabalho também faz uma vasta discussão sobre os desafios e as questões de pesquisa.

Embora os cinco trabalhos supracitados tenham feito revisões sobre a técnica de agrupamento de dados mistos, ainda não foi publicado nenhuma revisão sistemática da literatura para este tema. Uma revisão sistemática se preocupa a responder uma pergunta pontal sobre o tema em questão, beneficiando-se de um método regrado para encontrar e selecionar os trabalhos que estejam alinhados com o objetivo da análise, o que contribui com a transparência da pesquisa. Segundo Kitchenham (2004) a vantagem de uma revisão sistemática está em resumir as informações existentes sobre alguns fenômenos de maneira completa e imparcial, além de ser possível explorar os dados usando técnicas de meta-análise, aumentando a 
probabilidade de detectar efeitos reais que estudos individuais menores seriam incapazes de detectar.

Portanto, este artigo tem como objetivo realizar uma revisão sistemática da literatura sobre a técnica de agrupamento de dados mistos, especificamente sobre os trabalhos baseados no algoritmo k-means, que endereçaram uma função de dissimilaridade para cada tipo de atributo (numérico e categórico) e que foram publicados em veículos de divulgação científica de alto impacto. Para isso, almeja-se encontrar as palavras-chave que endereçam os trabalhos alinhados com o objetivo desta análise, definir a base de dados, critérios e filtros de seleção para que seja possível obter uma visão sobre a temática em questão. Este artigo está organizado como segue. A seção 2 descreve a metodologia utilizada nesta revisão bibliográfica sistemática, apresentando as etapas do trabalho. A seção 3 apresenta os resultados da revisão, trazendo uma análise quantitativa dos trabalhos publicados na temática de interesse, bem como uma breve descrição deles. Finalmente, a seção 4 conclui o trabalho.

\section{METODOLOGIA}

Uma das principais diferenças entre a revisão sistemática e a revisão tradicional, também conhecida por revisão narrativa, está no fato de que a primeira estabelece formalmente um protocolo, ou seja, um plano para conduzir a busca, seleção e discussão dos trabalhos. Dessa forma, o protocolo adotado nesta revisão se baseia nas etapas sugeridas por Kitchenham (2004), que consistem em definir: (i) as perguntas a serem respondidas; (ii) as palavras-chaves, bases de dados e a string de busca; (iii) os critérios de seleção dos trabalhos; (iv) a estratégia de exploração dos trabalhos, e $(v)$ a estratégia de síntese dos trabalhos selecionados. Considerando que o interesse central desta revisão é a técnica de agrupamento de dados mistos (apenas atributos numéricos e categóricos), especificamente os trabalhos baseados no algoritmo k-means, que endereçaram uma função de dissimilaridade para cada tipo de atributo e que foram publicados em veículos de divulgação cientifica de alto impacto, definiu-se cinco questões de interesse (Tabela 1), as quais serão respondidas na próxima seção, juntamente com a descrição e análise dos trabalhos selecionados.

Tabela 1. Questões de interesse desta revisão sistemática da literatura

\begin{tabular}{cl}
\hline Código & \multicolumn{1}{c}{ Questões } \\
\hline Q1 & Com que frequência esta temática é explorada? \\
Q2 & Quem são os principais autores e países que publicam neste tema? \\
Q3 & Quais são as áreas que mais publicam nesta temática? \\
Q4 & Quais são os trabalhos mais citados? \\
Q5 & Existem temas comumente abordados pelos trabalhos? \\
\hline
\end{tabular}

Levando em conta as questões da Tabela 1, realizou-se uma busca e um estudo prévio de trabalhos que endereçavam a técnica de agrupamento de dados mistos. Este estudo foi norteado pelo objetivo de conhecer os termos-chave mais utilizados e de compreender os conceitos básicos desta temática. Após isso, foi possível estabelecer as palavras-chaves e uni-las em uma string de busca, a qual foi usada na base de dados Scopus. A string foi definida como segue ((cluster* AND ("mixed data*" OR "mixed type" OR "mixed-type" OR "multiple attribute*" OR "mixed feature*" OR "mixed variable*" OR "numeric* and categoric*" OR "categoric* and numeric*" OR "numeric* and non-numeric*" OR "non-numeric* and numeric*" OR "internal and categoric*" OR "categoric* and internal")). A busca ocorreu no título, palavras-chave e resumo dos trabalhos indexados na base. A pesquisa ocorreu em agosto de 2019.

Como critérios de seleção dos trabalhos, estabeleceu-se que todos os trabalhos até 2018 seriam considerados na análise, embora apenas trabalhos publicados em inglês seriam passíveis de consideração, além daqueles que tivessem sido publicados em veículos de divulgação científica com o indicador Journal Citation Report (JCR) maior do que um, ou com pelo menos dois indicadores maiores do que um, considerando o JCR, CiteScore, SCImago Journal Rank (SJR) and Source Normalized Impact per Paper (SNIP) de 2019. Após a definição dos critérios de seleção, ainda foi estabelecido que os artigos passariam por três filtros de leitura a fim de selecionar apenas os trabalhos que estivessem alinhados com o objetivo da análise. O primeiro filtro consistiu na leitura do título, resumo e palavras-chave de todos os artigos considerados até então. Dos trabalhos que passavam pelo primeiro filtro, estabeleceu-se que a leitura deveria contemplar a introdução e conclusão dos trabalhos. Finalmente, no terceiro filtro foi feita a leitura do trabalho completo, de maneira atenta e detalhada. Como resultado do terceiro filtro, obteve-se os trabalhos analisados 
neste artigo, cuja apresentação e discussão são feitas na próxima seção. A Tabela 2 traz uma sumarização da quantidade de trabalhos que, primeiramente, retornou na base de dados, e permaneceram ao longo da revisão após a aplicação dos critérios e filtros de seleção.

Tabela 2. Sumarização dos resultados da revisão sistemática.

\begin{tabular}{ccccc}
\hline Base de dados & Total & em Inglês & Após os critérios & Após os filtros \\
\hline Scopus & 1.104 & 1.010 & 456 & 37 \\
\hline
\end{tabular}

Como pode ser visto na Tabela 2, um total de 1.104 trabalhos foram obtidos como resultado da busca pela string apresentada. Ao refinar a busca, restringindo-a os trabalhos em inglês, obteve-se um total de 1.010. Após a aplicação do critério que considerava o impacto do veículo de divulgação científica, 456 trabalhos permaneceram. Como resultado dos filtros, obteve-se 37 trabalhos que abordaram o agrupamento de dados mistos baseados no algoritmo k-means, entre os quais estavam trabalhos que propuseram novos algoritmos e trabalhos que apenas aplicaram algum algoritmo existente.

\section{RESULTADOS}

A seguir, os 37 trabalhos resultantes da revisão sistemática são brevemente descritos. Após isso, apresenta-se uma análise gráfica destes trabalhos e responde-se as questões da Tabela 1. O primeiro trabalho que estava alinhado com os objetivos desta revisão foi o de Huang (1998). O autor propões duas modificações no algoritmo $k$-means para que este pudesse trabalhar com dados categóricos ( $k$-modes) e com dados mistos (k-prototypes). Ng and Wong (2002) propuseram uma versão fuzzy para o k-modes e para o k-prototype, além de aplicarem o tabu search para a definição dos centroides. Ahmad and Dey (2007) propuseram uma nova função objetivo e uma nova forma de mensurar a dissimilaridade entre os registros baseada na co-ocorrência dos valores. Bushel, Wolfinger and Gibson (2007) apresentam o algoritmo modk-prototype, uma modificação do algoritmo k-prototype que lida com dados médicos, os quais consideram simultaneamente microarranjos de expressão génica, classes de variáveis fenotípicas conhecidas, como avaliações de química clínica e observações histopatológicas. Cheng and Leu (2008) propuseram um novo algoritmo baseado no k-prototype para lidar com as restrições impostas pelo problema de inspeção de manutenção de pontes.

Lee and Pedrycz (2009) apresentam um novo algoritmo baseado no k-prototype, chamado de $p$-mode prototype, o qual considera a frequência das classes dos atributos categóricos para determinar os centroides. Cheng and Leu (2009) generalizam a proposta feita no artigo de 2008, nomeando o algoritmo proposto de constrained k-prototypes (CKP). Ahmad and Dey (2011) propuseram uma modificação no $k$-means de maneira que o algoritmo conseguisse trabalhar com dados mistos em subespaços. Cheng and Leu (2011) generalizaram o método KJ, que é um método de diagrama de afinidade, para lidar dados mistos e restrições a fim de aplicá-lo para a classificação de defeitos de pontes. Os autores são os mesmos que propuseram o algoritmo CKP e que publicaram um artigo sobre o mesmo assunto em 2008. Pham, Suarez-Alvarez and Prostov (2011) aplicaram a meta-heurística Bee Algorithm no k-prototype com o objetivo de inicializar os centroides e evitar que o agrupamento ficasse preso em mínimos locais. Liang et al. (2012) determinaram o número de grupos pela entropia de informação no algoritmo k-prototype. Ji et al. (2013) propuseram uma nova função de dissimilaridade para o algoritmo k-prototype, além de um método para determinar o centroide. Cheung and Jia (2013) apresentaram um algoritmo chamado em Iterative Clustering Learning Based on Object-Cluster Similarity Metric (OCIL) que calcula o número de grupos, penalizando aqueles que são redundantes. Ovesná et al. (2013) analisaram a estrutura genética de uma coleção de cevada pelo agrupamento de dados usando o k-means e uma matriz de dissimilaridade construída pela dissimilaridade de Gower. Han et al. (2014) fizeram uma generalização do algoritmo k-annonumity para dados mistos. O algoritmo k-annonumity visa proteger a privacidade dos dados durante o processo de mineração. Harikumar and Surya (2015) propuseram uma modificação no k-medoid (algoritmo PAM) para trabalhar com dados mistos, por meio de uma nova função de dissimilaridade. Ji et al. (2015) apresentaram um método para inicialização dos centroides no algoritmo k-prototype. Sangam and Om (2015) propuseram o algoritmo Hybrid Data Labeling Algorithm (HDLA) para atribuir um novo registro a um dos grupos já existentes. Os autores usam os algoritmos k-prototype e o OCIL. Thong and Son (2016) fazem uma melhoria no Picture Fuzzy Set (PFS) por meio da definição de múltiplos centros usando o Particle Swarm Optimization (PSO). 
Ahmad and Hashmi (2016) fizeram uma generalização do algoritmo $k$-harmonic para dados mistos. O k-harmonic é um algoritmo para dados numéricos que propõe um método melhor para a inicialização dos centroides ao invés de fazer uma inicialização randômica no algoritmo k-means. Amorim and Makarenkov (2016) apresentaram uma modificação no k-means para dados mistos, atribuindo diferentes valores de peso para os atributos ao considerar um cenário de sub agrupamento. Bishnu and Bhattacherjee (2016) apresentaram uma generalização do k-modes para dados mistos com o objetivo de aplicar o algoritmo proposto em uma análise de custo de software. Drakopoulos et al. (2016) abordaram o problema de inicialização dos centroides no $k$-means para dados mistos por meio de algoritmos fuzzy que dividem o conjunto de dados em grids e pela densidade determinam o ponto de centroide. Além disso, o artigo também propõe um índice de qualidade externo de agrupamento para dados mistos.

Wangchamhan, Chiewchanwattana and Sunat (2017) endereçaram o trabalho para resolver o problema de inicialiação dos centróides no k-means, considerando a dissimilaridade de Gower para lidar com dados mistos. Kim (2017) propuseram um método para acelerar a execução do algoritmo $k$-prototype, pela redução do cálculo das distâncias. Özbakır and Turna (2017) modelaram duas meta-heurísticas para endereçar o problema de definição dos grupos. Bathla, Aggarwal and Rani (2018) propuseram a implementação do $k$-prototype no mapreduce para trabalhar com big data. Sangam and Om (2018) apresentaram uma nova função de dissimilaridade para o k-prototypes a fim de igualar as escalas de ambas dissimilaridades (categórica e numérica), sem que o algoritmo perdesse a escalabilidade. Davoodi and Moradi (2018) utilizaram as mesmas dissimilaridades definidas em Ji et al. (2012) para compor uma das etapas de um framework de deep learning com o objetivo de fazer a previsão de mortalidade em unidade de urgência. Gu et al. (2018) propuseram uma modificação do k-prototype para que o algoritmo fosse capaz de lidar com multiobjetivos e com a auto adaptação do número de cluster com objetivo de otimizar a combustão em uma cadeira, sujeita a restrições. Hong et al. (2018) apresentaram uma modificação na função de dissimilaridade do k-prototype com o objetivo de gerenciar o comportamento de carga de veículos elétricos. Jia and Cheung (2018) propuseram um algoritmo para atribuir peso para as variáveis nos diferentes grupos (sub agrupamento), além disso apresentaram um método de inicialização dos centroides e definição do número dos grupos. Li et al. (2018) desenvolveram um algoritmo para determinar o número de grupos, considerando um método de penalização para evitar grupos redundantes, além de um método para a inicialização dos centroides para realizar uma análise de eventos excepcionais em armazéns. Najjar et al. (2018) aplicaram o algoritmo k-prototype para analisar dados médicos. Sangam and Om (2018) generalizam o algoritmo HDLA para fluxo de dados. Yao et al. (2018) apresentaram um método para a inicialização dos centroides e atualização do número de grupos no k-prototype.

\subsection{Análise Gráfica e Respostas}

Com o objetivo de analisar visualmente os trabalhos resultantes da revisão sistemática, emitiu-se quatro gráficos. O Gráfico 1 (a) contempla a quantidade de trabalhos publicados por ano, o Gráfico 1 (b) traz a porcentagem de trabalhos por área do conhecimento, já os Gráficos 1 (c) e (d) mostram os dez principais autores e países que mais publicaram trabalhos na temática desta revisão, respectivamente. Considerando a descrição dos trabalhos e os gráficos, é possível responder algumas questões da Tabela 1. As outras informações que endereçam as respostas das questões remanescentes são fornecidas diretamente na resposta de cada uma. As respostas para cada questão seguem na Tabela 3.

\section{CONCLUSÃO}

Este trabalho explorou os algoritmos de agrupamento de dados mistos baseados no k-means, divulgados em veículos de divulgação científica de alto impacto e que atribuiu uma função de dissimilaridade para cada tipo de atributo (numérico e categórico). Como resultado, obteve-se uma visão holística do desenvolvimento desta temática e compreendeu-se que, embora o agrupamento de dados mistos seja um tema recentemente abordado pelos pesquisadores, sua aplicação é demandada por inúmeras áreas do conhecimento. 


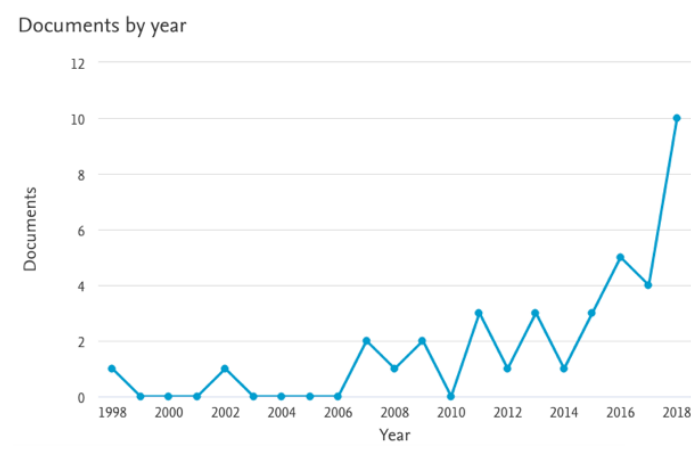

(a) Quantidade de trabalhos publicados por ano

Documents by author

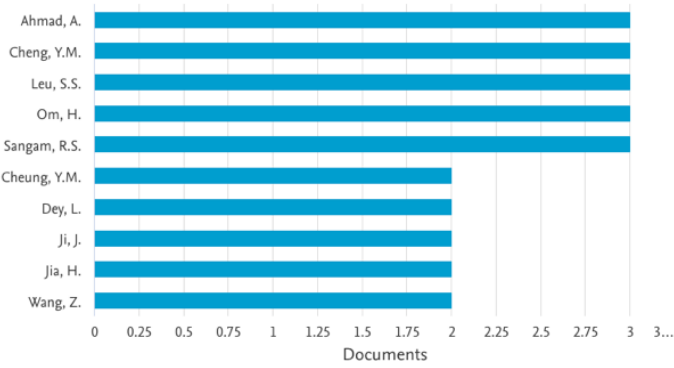

(c) Dez primeiros autores que mais publicaram
Documents by subject area

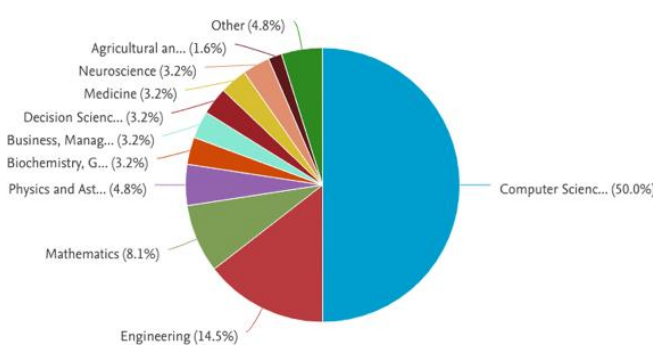

(b) Porcentagem de trabalhos por área de conhecimento

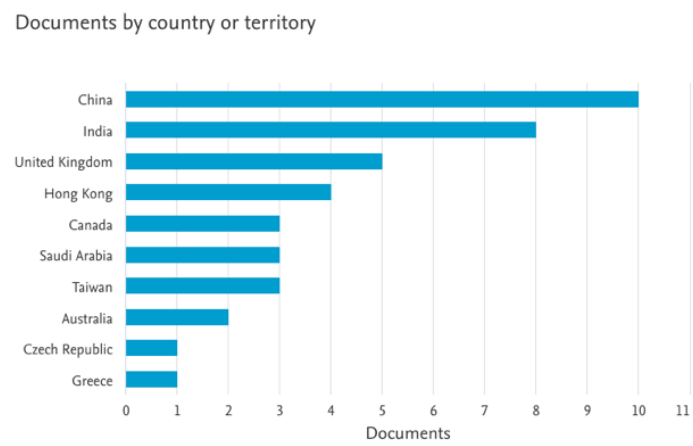

(d) Dez primeiros países que mais publicaram

Gráfico 1. Análise gráfica dos 37 artigos resultantes da revisão sistemática. Fonte: Scopus (2019)

Tabela 3. Respostas das questões de interesse desta revisão sistemática

\begin{tabular}{|c|c|}
\hline Código & Questões e respostas \\
\hline$Q 1$ & Com que frequência esta temática é explorada? \\
\hline $\mathrm{R} 1$ & $\begin{array}{l}\text { O Gráfico } 1 \text { (a) mostra que a frequência de publicação até } 2010 \text { foi de até dois artigos por ano. A partir de } \\
2011 \text { a quantidade de artigos por ano aumentou consideravelmente, mostrando que esta temática está em alta. }\end{array}$ \\
\hline$Q 2$ & Quem são os principais autores e países que publicam neste tema? \\
\hline $\mathrm{R} 2$ & $\begin{array}{l}\text { Os Gráficos } 1 \text { (c) e (d) trazem os dez principais autores e países que endereçaram seus trabalhos nesta } \\
\text { temática, respectivamente. Pode-se notar que os pesquisadores Ahmad, A., Cheng, Y. M., Leu, S. S. Om, H. e } \\
\text { Sangam, R. S. são os que mais publicaram trabalhos neste tema, seguidos de outros. Com relação aos países, } \\
\text { China, Índia e Reino Unido são os três principais países que contribuíram com essa temática, seguidos de } \\
\text { Hong Kong, Canada, Arábia Saudita, Taiwan, Austrália, República Checa e Grécia. }\end{array}$ \\
\hline Q3 & Quais são as áreas que mais publicam nesta temática? \\
\hline R3 & $\begin{array}{l}\text { O Gráfico } 1 \text { (b) mostra que as áreas que mais publicam são Ciência da Computação, Engenharia e } \\
\text { Matemática. }\end{array}$ \\
\hline$Q 4$ & Quais são os trabalhos mais citados? \\
\hline $\mathrm{R} 4$ & $\begin{array}{l}\text { Os três trabalhos mais citados são do Huang (1998), com } 1.168 \text { citações, seguido do artigo do Ahmad and } \\
\text { Dey (2007) com } 285 \text { citações e do trabalho de Ng and Wong (2002) com } 70 \text { citações. }\end{array}$ \\
\hline Q5 & Existem temas comumente abordados pelos trabalhos? \\
\hline R5 & $\begin{array}{l}\text { Sim, os trabalhos generalizam algoritmos tradicionais para dados numéricos para que estes possam lidar com } \\
\text { dados mistos. Além disso, é comum propostas para a definição do número de grupos e centroides. }\end{array}$ \\
\hline
\end{tabular}




\section{REFERÊNCIAS}

Ahmad, Amir and Dey, L. (2007) 'A k-mean clustering algorithm for mixed numeric and categorical data', Data and Knowledge Engineering, 63(2), pp. 503-527. doi: 10.1016/j.datak.2007.03.016.

Ahmad, A and Dey, L. (2007) 'A k-mean clustering algorithm for mixed numeric and categorical data', Data and Knowledge Engineering, 63(2), pp. 503-527. doi: 10.1016/j.datak.2007.03.016.

Ahmad, A. and Dey, L. (2011) 'A k-means type clustering algorithm for subspace clustering of mixed numeric and categorical datasets', Pattern Recognition Letters, 32(7), pp. 1062-1069. doi: 10.1016/j.patrec.2011.02.017.

Ahmad, A. and Hashmi, S. (2016) 'K-Harmonic means type clustering algorithm for mixed datasets', Applied Soft Computing Journal, 48, pp. 39-49. doi: 10.1016/j.asoc.2016.06.019.

Ahmad, A. and Khan, S. S. (2019) 'Survey of State-of-the-Art Mixed Data Clustering Algorithms', IEEE Access, 7, pp. 31883-31902. doi: 10.1109/ACCESS.2019.2903568.

Amorim, R. C. de and Makarenkov, V. (2016) 'Applying subclustering and L $<$ inf $>\mathrm{p}</$ inf $>$ distance in Weighted K-Means with distributed centroids', Neurocomputing, 173, pp. 700-707. doi: 10.1016/j.neucom.2015.08.018.

Balaji, K. and Lavanya, K. (2018) 'Clustering Algorithms for Mixed Datasets: A Review', International Journal of Pure and Applied Mathematics, 118(7), pp. 547-556.

Bishnu, P. S. and Bhattacherjee, V. (2016) 'Software cost estimation based on modified K-Modes clustering Algorithm', Natural Computing, 15(3), pp. 415-422. doi: 10.1007/s11047-015-9492-7.

Bushel, P. R., Wolfinger, R. D. and Gibson, G. (2007) 'Simultaneous clustering of gene expression data with clinical chemistry and pathological evaluations reveals phenotypic prototypes', BMC Systems Biology, 1, pp. 1-20.

Cheng, Y.-M. and Leu, S.-S. (2008) 'Constraint-based clustering model for determining contract packages of bridge maintenance inspection', Automation in Construction, 17(6), pp. 682-690. doi: 10.1016/j.autcon.2007.12.001.

Cheng, Y.-M. and Leu, S.-S. (2009) 'Constraint-based clustering and its applications in construction management', Expert Systems with Applications, 36(3 PART 2), pp. 5761-5767. doi: 10.1016/j.eswa.2008.06.100.

Cheng, Y. M. and Leu, S. Sen (2011) 'Integrating data mining with KJ method to classify bridge construction defects', Expert Systems with Applications. Elsevier Ltd, 38(6), pp. 7143-7150. doi: 10.1016/j.eswa.2010.12.047.

Cheung, Y.-M. and Jia, H. (2013) 'Categorical-and-numerical-attribute data clustering based on a unified similarity metric without knowing cluster number', Pattern Recognition, 46(8), pp. 2228-2238. doi: 10.1016/j.patcog.2013.01.027.

Davoodi, R. and Moradi, M. H. (2018) 'Mortality prediction in intensive care units (ICUs) using a deep rule-based fuzzy classifier', Journal of Biomedical Informatics, 79, pp. 48-59. doi: 10.1016/j.jbi.2018.02.008.

Drakopoulos, G. et al. (2016) ‘A Fuzzy Graph Framework for Initializing k-Means', International Journal on Artificial Intelligence Tools, 25(6). doi: 10.1142/S0218213016500317.

Foss, A. H., Markatou, M. and Ray, B. (2019) 'Distance Metrics and Clustering Methods for Mixed-type Data', International Statistical Review. John Wiley \& Sons, Ltd (10.1111), 87(1), pp. 80-109. doi: 10.1111/insr.12274.

$\mathrm{Gu}$, H. et al. (2018) 'Optimized scheme in coal-fired boiler combustion based on information entropy and modified K-prototypes algorithm', Results in Physics, 9, pp. 1262-1274. doi: 10.1016/j.rinp.2018.04.045.

Han, J. et al. (2014) 'MAGE: A semantics retaining K-anonymization method for mixed data', Knowledge-Based Systems. Elsevier B.V., 55, pp. 75-86. doi: 10.1016/j.knosys.2013.10.009.

Harikumar, S. and Surya, P. V (2015) 'K-Medoid Clustering for Heterogeneous DataSets', in Procedia Computer Science, pp. 226-237. doi: 10.1016/j.procs.2015.10.077.

Hennig, C. (2015) 'What are the true clusters?', Pattern Recognition Letters, 64, pp. 53-62. Available at: http://arxiv.org/abs/1502.02555 (Accessed: 6 August 2019).

Hong, J. et al. (2018) 'Development of EV charging templates: An improved K-prototypes method', IET Generation, Transmission and Distribution, 12(20), pp. 4361-4367. doi: 10.1049/iet-gtd.2017.1911.

Huang, Z. (1998) 'Extensions to the k-means algorithm for clustering large data sets with categorical values', Data Mining and Knowledge Discovery, 2(3), pp. 283-304. doi: 10.1023/A:1009769707641.

Jain, A. K. (2010) 'Data clustering: 50 years beyond K-means', Pattern Recognition Letters. Elsevier B.V., 31(8), pp. 651-666. doi: 10.1016/j.patrec.2009.09.011.

Jain, A. K. and Dubes, R. C. (1988) Algorithms for clustering data. Englewood Cliffs, N.J., USA: Prentice Hall.

Ji, J. et al. (2012) 'A fuzzy k-prototype clustering algorithm for mixed numeric and categorical data', Knowledge-Based Systems. Elsevier, 30, pp. 129-135. doi: 10.1016/J.KNOSYS.2012.01.006.

Ji, J. et al. (2013) 'An improved k-prototypes clustering algorithm for mixed numeric and categorical data', Neurocomputing, 120, pp. 590-596. doi: 10.1016/j.neucom.2013.04.011. 
Ji, J. et al. (2015) 'An Initialization Method for Clustering Mixed Numeric and Categorical Data Based on the Density and Distance', International Journal of Pattern Recognition and Artificial Intelligence, 29(7).

Jia, H. and Cheung, Y.-M. (2018) 'Subspace clustering of categorical and numerical data with an unknown number of clusters', IEEE Transactions on Neural Networks and Learning Systems, 29(8), pp. 3308-3325. doi: 10.1109/TNNLS.2017.2728138.

Kim, B. (2017) 'A fast K-prototypes algorithm using partial distance computation', Symmetry, 9(4). doi: 10.3390/sym9040058.

Kitchenham, B. (2004) Procedures for Performing Systematic Literature Reviews, Joint Technical Report, Keele University TR/SE-0401 and NICTA TR-0400011T.1.

Lee, M. and Pedrycz, W. (2009) 'The fuzzy C-means algorithm with fuzzy P-mode prototypes for clustering objects having mixed features', Fuzzy Sets and Systems, 160(24), pp. 3590-3600. doi: 10.1016/j.fss.2009.06.015.

Li, N. et al. (2018) 'Exceptional events classification in warehousing based on an integrated clustering method for a dataset with mixed-valued attributes', International Journal of Computer Integrated Manufacturing, 31(11), pp. 1078-1096. doi: 10.1080/0951192X.2018.1509129.

Liang, J. et al. (2012) 'Determining the number of clusters using information entropy for mixed data', Pattern Recognition. Elsevier, 45(6), pp. 2251-2265. doi: 10.1016/j.patcog.2011.12.017.

MacQueen, J. (1967) 'Some Methods for Classification and Analysis of Multivariate Observations', in 5th Berkeley Symposium on Mathematical Statistics and Probability. Berkeley, CA, USA: University of California Press, pp. 281-297.

Miyamoto, S., Huynh, V.-N. and Fujiwara, S. (2018) 'Methods for Clustering Categorical and Mixed Data: An Overview and New Algorithms', Proceedings of the International Symposium on Integrated Uncertainty in Knowledge Modelling and Decision Making (IUKM 2011), 1, pp. 75-86. doi: 10.1007/978-3-319-75429-1_7.

Najjar, A. et al. (2018) 'A two-step approach for mining patient treatment pathways in administrative healthcare databases', Artificial Intelligence in Medicine, 87, pp. 34-48. doi: 10.1016/j.artmed.2018.03.004.

$\mathrm{Ng}, \mathrm{M}$. K. and Wong, J. C. (2002) 'Clustering categorical data sets using tabu search techniques', Pattern Recognition, 35(12), pp. 2783-2790. doi: 10.1016/S0031-3203(02)00021-3.

Ovesná, J. et al. (2013) 'Analysis of the Genetic Structure of a Barley Collection Using DNA Diversity Array Technology (DArT)', Plant Molecular Biology Reporter, 31(2), pp. 280-288. doi: 10.1007/s11105-012-0491-x.

Özbakır, L. and Turna, F. (2017) 'Clustering performance comparison of new generation meta-heuristic algorithms', Knowledge-Based Systems, 130, pp. 1-16. doi: 10.1016/j.knosys.2017.05.023.

Pham, D.-T., Suarez-Alvarez, M. M. and Prostov, Y. I. (2011) 'Random search with k-prototypes algorithm for clustering mixed datasets', Proceedings of the Royal Society A: Mathematical, Physical and Engineering Sciences, 467(2132), pp. 2387-2403. doi: 10.1098/rspa.2010.0594.

Sangam, R. S. and Om, H. (2015) 'Hybrid data labeling algorithm for clustering large mixed type data', Journal of Intelligent Information Systems, 45(2), pp. 273-293. doi: 10.1007/s10844-014-0348-X.

Sangam, Ravi Sankar and Om, H. (2018) 'An equi-biased k-prototypes algorithm for clustering mixed-type data', Sadhana - Academy Proceedings in Engineering Sciences. Springer India, 43(3), pp. 1-12. doi: 10.1007/s12046-0180823-0.

Sangam, R S and Om, H. (2018) 'Equi-Clustream: A framework for clustering time evolving mixed data', Advances in Data Analysis and Classification, 12(4), pp. 973-995. doi: 10.1007/s11634-018-0316-3.

Scopus (2019) Scopus, Analyze search results. Available at: https://www.scopus.com/.

Thong, P. H. and Son, L. H. (2016) 'Picture fuzzy clustering for complex data', Engineering Applications of Artificial Intelligence, 56, pp. 121-130. doi: 10.1016/j.engappai.2016.08.009.

van de Velden, M., D'Enza, A. I. and Markos, A. (2019) 'Distance-based clustering of mixed data', Wiley Interdisciplinary Reviews: Computational Statistics, 11(3). doi: 10.1002/wics.1456.

Wangchamhan, T., Chiewchanwattana, S. and Sunat, K. (2017) 'Efficient algorithms based on the k-means and Chaotic League Championship Algorithm for numeric, categorical, and mixed-type data clustering', Expert Systems with Applications, 90, pp. 146-167. doi: 10.1016/j.eswa.2017.08.004.

Xu, R. and Wunsch, D. C. (2009) Clustering. Edited by D. B. Fogel. Hoboken, New Jersey: John Wiley \& Sons, Inc.

Yao, X. et al. (2018) 'An Improved Clustering Algorithm and Its Application in WeChat Sports Users Analysis', in Procedia Computer Science, pp. 166-174. doi: 10.1016/j.procs.2018.03.067. 